\title{
Incidence of human granulocytic anaplasmosis in returning travellers with fever
}

\author{
Daniel Camprubí-Ferrer, MD ${ }^{1}$,* , Aránzazu Portillo, PhD², Sonia Santibáñez, PhD², \\ Alejandro Almuedo-Riera, MD' ${ }^{1}$, Natalia Rodriguez-Valero, MD ${ }^{1}$, Carme Subirà, BSc ${ }^{1}$, \\ Miguel J. Martinez, PhD' ${ }^{1}$, Jessica Navero-Castillejos, BSc ${ }^{1}$, Marc Fernandez-Pardos, \\ MSc $^{1}$, Blaise Genton, $\mathrm{PhD}^{3,4}$, Ludovico Cobuccio, MD ${ }^{3,4}$, Steven Van Den Broucke, \\ $\mathrm{MD}^{5}$, Emmanuel Bottieau, $\mathrm{PhD}^{5}$, Jose Muñoz, $\mathrm{PhD}^{1, \dagger}$ and José Antonio Oteo, $\mathrm{PhD}^{2, \dagger}$
} \footnotetext{
Switzerland and ${ }^{5}$ Institute of Tropical Medicine, 2000 Antwerp, Belgium

*To whom correspondence should be addressed. Tel: +34 93227 18 52; Email: dcamprub@clinic.cat

${ }^{\dagger}$ These authors contributed equally to this work.

Submitted 8 February 2021; Revised 23 March 2021; Accepted 31 March 2021
}

${ }^{1}$ ISGlobal, Hospital Clínic-Universitat de Barcelona, 08036 Barcelona, Spain, ${ }^{2}$ Center of Rickettsiosis and Arthropod-Borne Diseases, Hospital Universitario San Pedro-CIBIR, 26006 Logroño, Spain, ${ }^{3}$ Swiss Tropical and Public Health Institute, 4051 Basel, Switzerland, ${ }^{4}$ Center for Primary Care and Public Health, University of Lausanne, 1010,

\begin{abstract}
Background: Although tick-borne pathogens have been reported as an important cause of imported fever, the incidence of Anaplasma phagocytophilum, the causative agent of human granulocytic anaplasmosis (HGA), in travellers is unknown.

Methods: We conducted a prospective cohort study to investigate the aetiologies of fever in returning travellers (November 2017-July 2019). Polymerase chain reaction for msp2 gene amplification and indirect immunofluorescence assay for $A$. phagocitophilum were performed in all returning travellers with undifferentiated non-malarial fever.

Results: Among 141 travellers included, 8 patients were diagnosed with probable or confirmed HGA. The overall incidence rate of HGA was 19.9 cases/1000 person-week of travel. The main destination of travel was Asia, accounting for $62.5 \%$ patients with HGA. Co-infections were found in $37.5 \%$ of patients with HGA.

Conclusions: Diagnosis of HGA and empirical treatment with doxycycline should be considered in travellers with fever.
\end{abstract}

Key words: Anaplasma phagocytophilum, fever, travel, ticks, doxycycline, emerging infections, vector-borne diseases

\section{Introduction}

Coronavirus disease 2019 (COVID-19) pandemic has brought out the difficulties of facing the diagnosis and treatment of emerging infections, especially those presenting with imported fever. ${ }^{1}$ Febrile illness is estimated to occur in about $2-3 \%$ of returning travellers and accounts for up to $25 \%$ of patients seeking medical care in travel clinics. ${ }^{2,3}$ After ruling out common diseases such as malaria or arboviral infections, a large number of undifferentiated fever cases remain undiagnosed. ${ }^{4}$ Recently, some studies performed in endemic areas showed that pathogens transmitted by ticks or mites such as Orientia tsutsugamushi and several species of Rickettsia spp. must be considered as causative agents in non-malarial febrile illnesses. ${ }^{5}$

Anaplasma phagocytophilum is an obligate, intracellular Gram-negative bacteria transmitted by hard ticks from the family Ixodidae. It is the aetiological agent of Human Granulocytic Anaplasmosis (HGA), usually presenting as an undifferentiated febrile illness, with headache, myalgias/arthralgia and 
cytopenias. ${ }^{6}$ HGA can range from an asymptomatic selflimiting infection to a multi-organ failure fatal disease, requiring hospitalization in one-half of cases. ${ }^{6,7}$

HGA has mainly been described in America and Europe but, like other rickettsial infections, it seems to be severely underreported. ${ }^{6}$ Besides factors such as climate change, the increase of international travels has been suggested as an important cause of the emergence of tick-borne infections. ${ }^{7-9}$ Although HGA has been occasionally reported in febrile travellers, ${ }^{10}$ incidence of HGA among returning travellers is unknown.

\section{Methods}

We performed a prospective cohort study in patients presenting with fever within 28 days after an international trip at the Hospital Clinic of Barcelona (HCB), Spain, from November 2017 to July 2019. Inclusion criteria were temperature $\geq 37.5^{\circ} \mathrm{C}$ or feverish sensation accompanied by 2 of the 3 following symptoms: arthralgia, myalgia or chills. Patients younger than 18 years old and those requiring admission in a high-level isolation unit were excluded. In order to rule out malaria, a blood smear was systematically performed in all febrile travellers or migrants returning from malaria endemic areas. Patients presenting with $\geq 3$ stools per day, prominent cough, expectoration or dyspnea, urinary tract infection symptoms or signs consistent with skin or soft tissue infections were considered as differentiated fevers and were not eligible to participate. By contrary, febrile patients, presenting with no clear source of infection in whom malaria was ruled out, were defined as undifferentiated non-malarial fever (UNMF) cases and were eligible to participate.

In addition to the microbiological tests performed routinely (Table 1), blood samples at first visit and 28 days (range: 21-72) later were obtained in study participants. Polymerase chain reaction (PCR) for $m s p 2$ gene amplification in acute-phase whole blood or plasma samples and indirect immunofluorescence assay (IFA) for A. phagocitophylum (Focus Diagnostics, Cypress, CA, USA) in paired sera were performed. ${ }^{6,11-13}$ In patients diagnosed with HGA (either by IFA or PCR), PCRs and serologies for spotted fever group Rickettsia spp., typhus group Ricketssia spp., O. tsutsugamushi, Coxiella burnetii, Bartonella quintana, Bartonella benselae and serology for Borrelia burgdorferi were systematically performed in order to rule out possible cross-reactions and/or co-infections. Based on microbiological and clinical criteria, according to guidelines, ${ }^{6,12}$ and after expert discussion, patients were classified as confirmed HGA, probable HGA or cases without active HGA (Table 1). Cases with stable titres of $A$. phagocytophilum antibodies in acute and convalescent sera and titres $>4$-fold above the cut-off value were classified as probable cases when they presented with other confirmed infections. Possible co-infections were classified according to the scheme proposed by Phommarone et al. ${ }^{14}$

Overall incidence of HGA was presented as an incidence rate (IR) of confirmed and probable cases. Period at risk for HGA was considered as the time from the beginning of the trip until the onset of fever in participants diagnosed with HGA presenting with fever during the trip and as the overall duration of travel in the others. For the IR calculation, long trips ( $\geq 6$ months), expatriates and long term residents were excluded from the analysis.
Categorical variables are presented in percentages and quantitative variables are presented as median and interquartile ranges (IQR). Fisher's exact test was used to compare categorical variables between groups, while Mann-Whitney U test was used for quantitative variables. The statistical analysis was carried out using Stata 15 (StataCorp.2017).

The study was conducted following the principles of the Declaration of Helsinki. The ethical committee of the HCB approved the study (HCB/2017/0612). Informed written consent was given by all participants.

\section{Results}

\section{Incidence of HGA among febrile returning travellers}

During the study period, 141 patients with available paired sera out of a total of 179 patients with UNMF were included in the study. Of them, a total of 8 patients were diagnosed with probable or confirmed HGA, showing an overall IR of HGA of 19.9 cases 1000 person-week of travel (95\% CI: 9.6-41.5). Figure 1 illustrates the microbiological results of participants with HGA.

Two participants fulfilled the criteria for confirmed HGA. One had a seroconversion with no alternative diagnosis. The other confirmed case was diagnosed both by seroconversion and amplification of $m s p 2$ gene, with subsequent A.phagocitophilum sequencing. Based on confirmed cases, minimum IR of HGA is estimated to be 5.7 cases/1000 person-week of travel (95\% CI: 1.4-22.6).

Six participants fulfilled the criteria for probable HGA. Two of them presented seroconversion for A. phagocitophylum and an alternative diagnosis. The other 4 participants presented with high positive IFA titers $(\geq 64)$ in acute and convalescent sera or $<4$-fold elevation of IFA titers for A. phagocitophylum. Then, IR of probable HGA was estimated to be 14.2 cases/1000 person with UNMF-week of travel (95\%CI: 6.0-34.0). Data on incidence of probable and confirmed HGA are summarized in Table 1.

Among the 141 patients with UNMF included, positive IFA for A. phagocytophilum was detected in an additional 9 participants (17 patients in total), showing a global seroprevalence of $A$. phagocytophilum IgG antibodies of $12.1 \%$ (95\%CI: 7.2-18.6).

\section{Epidemiological and clinical characteristics of travellers diagnosed with HGA}

Table 2 describes the epidemiological and clinical features of the 8 travellers with HGA. The main destination of travel was Asia, accounting for 5 cases $(62.5 \%)$. Indeed, the two confirmed cases of HGA came from Thailand and Malaysia. All of them visited rural areas. Three $(37.5 \%)$ patients presented a clear risk of tick exposure: two of them had contact with animals and only one referred a tick bite, but none of them presented with an eschar on physical exam. The maximal incubation period (measured from the last day of the trip) was 6 days. All patients presented fever within the first 24 hours of symptoms' onset. Other common symptoms were arthralgia, myalgia, malaise, headache and rash. Common laboratory abnormalities were cytopenias $(75.0 \%)$ and 
Table 1. Incidence and definition criteria of confirmed and probable HGA

\begin{tabular}{|c|c|c|}
\hline & Confirmed HGA & Probable HGA $^{\mathrm{a}}$ \\
\hline Case definition & $\begin{array}{l}\text { Compatible clinical presentation AND: (i) positive PCR } \\
\text { assay and A. phagocytophilum sequencing OR } \\
\text { (ii) seroconversion or } \geq 4 \text {-fold increase of IFA titers with } \\
\text { no alternative confirmed diagnosis.b }\end{array}$ & $\begin{array}{l}\text { Compatible clinical presentation AND: } \\
\text { (i) seroconversion or } \geq 4 \text {-fold increase of IFA titers with } \\
\text { an alternative confirmed diagnosis }{ }^{\text {a }} \text { OR } \\
\text { (ii) high positive IFA titers }(\geq 64) \text { in acute and } \\
\text { convalescent sera or }<4 \text {-fold elevation of IFA titers } \\
\text { with no alternative diagnosis. }^{\text {b }}\end{array}$ \\
\hline $\operatorname{IR}^{\mathrm{c}}$ & 5.7 cases $/ 1000$ person with UNMF-week of travel & 14.2 cases $/ 1000$ person with UNMF—week of travel \\
\hline $95 \%$ CI & $1.4-22.6$ cases $/ 1000$ person with UNMF-week of travel & $6.0-34.0$ cases $/ 1000$ person with UNMF-week of travel \\
\hline
\end{tabular}

CI: confidence interval. IFA: indirect immunofluorescence assay.

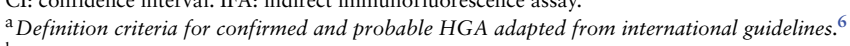

${ }^{\mathrm{b}}$ PCRs and serologies against dengue virus, chikungunya virus and Zika virus were routinely performed in all patients presenting with fever $\leq 14$ days after the trip. PCRs and serologies for spotted fever group Rickettsia spp., typhus group Ricketssia spp., O. tsutsugamushi, C. burnetii, B. quintana, B. henselae and serology for B. burgdorferi were systematically performed in patients diagnosed with HGA. Tests looking for other infections such as leptospirosis or HIV were performed based on clinical suspicion and risk exposures.

${ }^{\mathrm{c}}$ Long trips ( $\geq 6$ months), expatriates and long-term residents excluded.

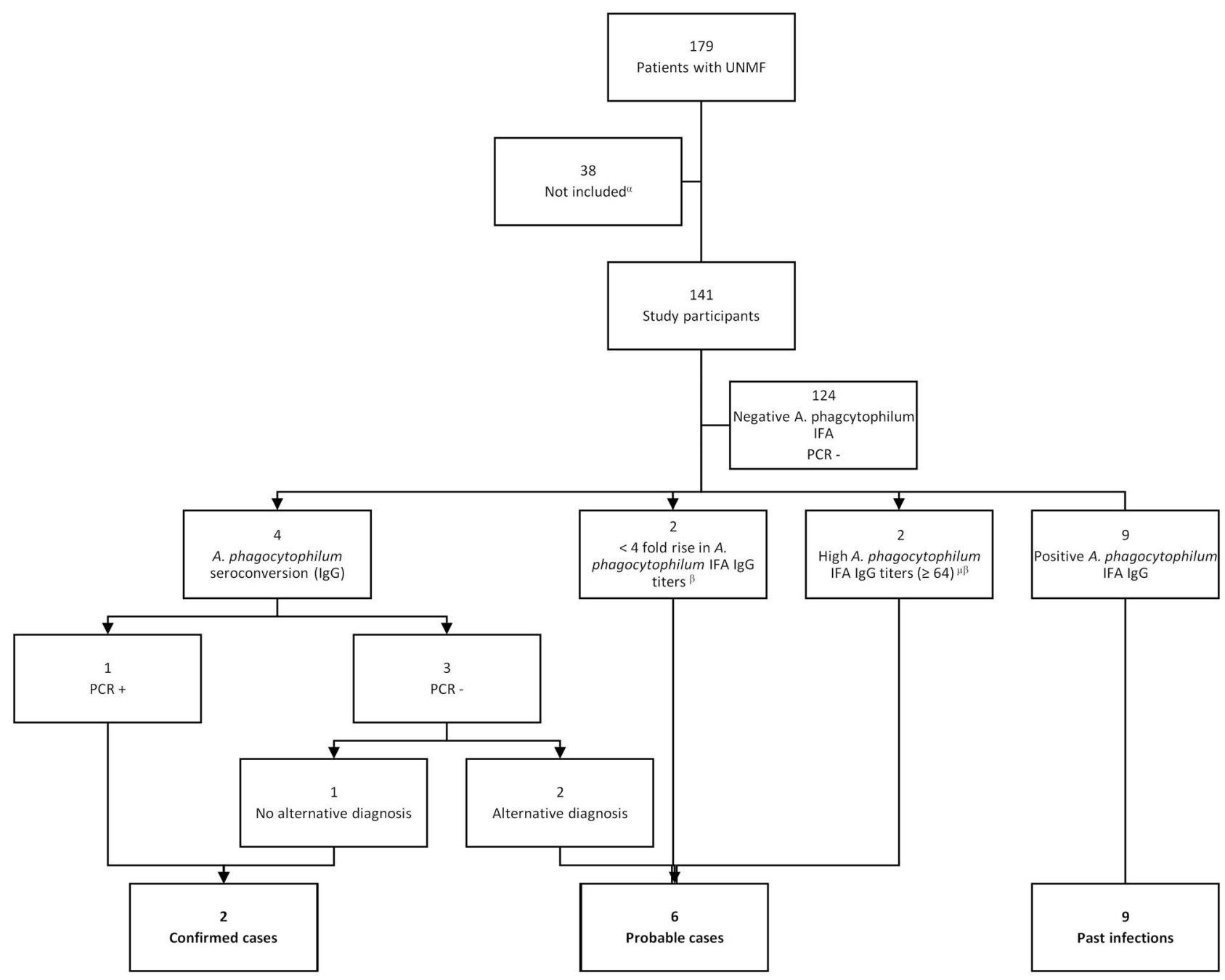

Figure 1. Flowchart of study participants with HGA.

IFA: indirect immunofluorescence assay. IgG: immunoglobulin G. ${ }^{\alpha} 38$ patients with no convalescent serum sample were excluded from the study $\beta$ Without an alternative diagnosis ${ }^{\mu} \mathrm{In}$ acute and convalescent sera. ${ }^{\pi}$ Not fulfilling criteria of previous categories.

mild elevation of C-reactive protein $(62.5 \%)$. The aforementioned clinical and demographic characteristics did not differ between travellers with HGA and those without HGA (Table 2).
Although only $2(25.0 \%)$ patients received an adequate antibiotic treatment (doxycycline $100 \mathrm{mg}$ bid for 7-14 days), fever disappeared in less than 5 days in 6 patients $(75.0 \%)$. 
Table 2. Comparison of epidemiological and clinical characteristics of travellers with and without HGA

\begin{tabular}{|c|c|c|c|c|c|}
\hline & $\begin{array}{l}\text { Confirmed HGA } \\
(n=2)\end{array}$ & $\begin{array}{l}\text { Probable HGA } \\
(n=6)\end{array}$ & $\begin{array}{l}\text { Overall HGA } \\
(n=8)\end{array}$ & $\begin{array}{l}\text { Non-HGA } \\
\text { undifferentiated fevers } \\
(n=133)\end{array}$ & $P$-value ${ }^{a}$ \\
\hline Male sex, $n / N(\%)$ & $1 / 2(50.0)$ & $3 / 6(50.0)$ & $4 / 8(50.0)$ & $71 / 133(53.4)$ & $>0.999^{\mathrm{b}}$ \\
\hline Age (years), Md (IQR) & $40.5(39-42)^{\mathrm{c}}$ & $26(26-53)$ & $32.5(26-47.5)$ & $34(28-43)$ & $0.954^{\mathrm{d}}$ \\
\hline Travel region, $n / \mathrm{N}(\%)$ & & & & & $0.938^{\mathrm{b}}$ \\
\hline - Asia/Southeast Asia ${ }^{\mathrm{e}}$ & $2 / 2(100)$ & $3 / 6(50.0)$ & $5 / 8(62.5)$ & $61 / 132(46.2)$ & \\
\hline - Africa $^{\mathrm{f}}$ & $0 / 2$ & $2 / 6(33.3)$ & $2 / 8(25.0)$ & $33 / 132(25.0)$ & \\
\hline - America ${ }^{g}$ & $0 / 2$ & $1 / 6(16.7)$ & $1 / 8(12.5)$ & $29 / 132(22.0)$ & \\
\hline - Europe & $0 / 2$ & $0 / 6$ & $0 / 8$ & $9 / 132(6.8)$ & \\
\hline Duration of travel (days), Md (IQR) & $16.5(16-17)$ & $15.5(13-16)$ & $16(14-16.5)$ & $17(12-26)$ & $0.688^{\mathrm{d}}$ \\
\hline \multicolumn{6}{|l|}{ Risk factors, $n / N(\%)$} \\
\hline - Rural areas & $2 / 2(100)$ & $6 / 6(100)$ & $8 / 8(100)$ & $118 / 133(88.7)$ & $0.600^{\mathrm{b}}$ \\
\hline - Animal contact & $1 / 2(50.0)$ & $1 / 6(16.7)$ & $2 / 8(25.0)$ & $58 / 133(43.6)$ & $0.467^{\mathrm{b}}$ \\
\hline - Tick bite & $0 / 2$ & $1 / 6(16.7)$ & $1 / 8(12.5)$ & $18 / 133(13.5)$ & $>0.999^{\mathrm{b}}$ \\
\hline Days of fever, Md (IQR) & $3.5(3-4)^{\mathrm{c}}$ & $6(2-6)$ & $4(2-6)$ & $5(3-7)$ & $0.864^{\mathrm{d}}$ \\
\hline \multicolumn{6}{|l|}{ Symptoms and signs, $n / N(\%)$} \\
\hline - Fever & $2 / 2(100)$ & $6 / 6(100)$ & $8 / 8(100)$ & $133 / 133(100)$ & - \\
\hline - Malaise/fatigue & $1 / 2(50.0)$ & $5 / 6(83.3)$ & $6 / 8(75.0)$ & $105 / 133(78.9)$ & $0.678^{\mathrm{b}}$ \\
\hline - Headache & $1 / 2(50.0)$ & $5 / 6(83.3)$ & $6 / 8(75.0)$ & $99 / 133(74.4)$ & $>0.999^{\mathrm{b}}$ \\
\hline - Arthralgia/myalgia & $2 / 2(100)$ & $3 / 6(50.0)$ & $5 / 8(62.5)$ & $97 / 133(72.9)$ & $0.685^{\mathrm{b}}$ \\
\hline - Rash & $2 / 2(100)$ & $2 / 6(33.3)$ & $4 / 8(50.0)$ & $54 / 133(40.6)$ & $0.717^{\mathrm{b}}$ \\
\hline - Eschar & $0 / 2$ & $0 / 6$ & $0 / 9$ & $11 / 133(8.3)$ & $>0.999^{b}$ \\
\hline \multicolumn{6}{|l|}{ Blood tests, $n / N(\%)$} \\
\hline - Any cytopenia & $1 / 2(50.0)$ & $5 / 6(83.3)$ & $6 / 8(75.0)$ & $82 / 131(62.6)$ & $0.710^{\mathrm{b}}$ \\
\hline - Leukopenia & $1 / 2(50.0)$ & $3 / 6(50.0)$ & $3 / 8(37.5)$ & $40 / 131(30.5)$ & $0.703^{\mathrm{b}}$ \\
\hline - Neutropenia & $1 / 2(50.0)$ & $3 / 6(50.0)$ & $4 / 8(50.0)$ & $49 / 131(37.4)$ & $0.480^{\mathrm{b}}$ \\
\hline - Lymphopenia & $1 / 2(50.0)$ & $4 / 6(66.7)$ & $5 / 8(62.5)$ & $55 / 131(42.0)$ & $0.255^{\mathrm{b}}$ \\
\hline - Thrombocytopenia & $1 / 2(50.0)$ & $0 / 6$ & $1 / 8(12.5)$ & $18 / 130(13.9)$ & $>0.999^{\mathrm{b}}$ \\
\hline - Anemia & $0 / 2$ & $1 / 6(16.7)$ & $1 / 8(12.5)$ & $11 / 129(8.53)$ & $0.529^{\mathrm{b}}$ \\
\hline - Elevated CRP & $1 / 2(50.0)$ & $4 / 6(66.7)$ & $5 / 8(62.5)$ & $81 / 133(60.9)$ & $>0.999^{\mathrm{b}}$ \\
\hline - Elevated transaminases & $1 / 2(50.0)$ & $2 / 6(33.3)$ & $3 / 8(37.5)$ & $68 / 133(51.1)$ & $0.493^{\mathrm{b}}$ \\
\hline Hospital admission, $n / N(\%)$ & $0 / 2$ & $1 / 6(16.7)$ & $1 / 8(12.5)$ & $26 / 133(19.5)$ & $>0.999^{\mathrm{b}}$ \\
\hline Cure at 30 days, $n / N(\%)$ & $2 / 2(100)$ & $6 / 6(100)$ & $8 / 8(100)$ & $101 / 133(75.9)$ & $0.198^{\mathrm{b}}$ \\
\hline
\end{tabular}

CPR: C-reactive protein. Md: Median. n: number of cases. N: total number of patients diagnosed with HGA

${ }^{a}$ Comparison between overall HGA and non-HGA undifferentiated fever groups.

${ }^{b}$ Fisher's exact test.

${ }^{\mathrm{c}}$ Range.

${ }^{\mathrm{d}}$ Wilcoxon rank-sum test.

${ }^{\mathrm{e}}$ Countries visited in Asia included Thailand, Vietnam, Singapore, Malaysia, Indonesia and India.

${ }^{\mathrm{f}}$ Countries visited in Africa included Madagascar and Angola.

The visited country in the America was Costa Rica.

Only one patient required hospital admission. One month after inclusion, all patients but one were completely asymptomatic. The only patient that was still symptomatic was diagnosed with myelodysplastic syndrome; symptoms after 1 month were not considered any more as related to A. phagocytophilum infection.

\section{Co-infections}

Three out of 8 HGA participants $(37.5 \%)$ had confirmed, probable or possible co-infections with Chikungunya, Dengue and C. burnetii. Supplementary Table 1 shows detailed microbiological information of HGA co-infections.

\section{Discussion}

The main finding of our study is an overall IR of HGA of 19.9 cases/1000 person with UNMF-week of travel, that means that patients presenting with fever after an international travel have an increased risk of HGA of almost $2 \%$ for every week of travel. Although there is a scarcity on the available published information about the incidence of HGA in travellers, our study shows that the incidence of HGA among returning travellers with fever is not negligible. In addition, the incidence of vectorborne infections is expected to increase due to recent lockdowns and breakdowns in control strategies in endemic areas due to COVID-19 pandemic. $^{15}$ As with some other neglected diseases, the non-specific presentation of HGA, the low awareness of this infection by physicians and the lack of access to diagnostic tools in several centers can explain why HGA is currently underreported..$^{6-8,16}$ Moreover, since two patients had stable antibody levels against $A$. phagocytophilum, we cannot completely rule out that these patients acquired the infection in Spain before travelling. This is not the case for the remaining patients. Time since the last day of the trip to the onset of fever was below 5 days 
in all but one case, and time since the beginning of the trip to the onset of fever was consistent with the usual median HGA incubation period of 11 days (range 5-21). ${ }^{6}$ The only patient who presented with fever 6 days after the last day of the trip denied any exposure or risk factor for HGA since the arrival, making very unlikely the acquisition of HGA in Spain.

Most cases of HGA have been previously reported in North America and Europe. ${ }^{7,8}$ Nevertheless, in our study, A. phagocytophilum infection was commonly found in travellers from Asia, where A. phagocytophilum is increasingly been reported. ${ }^{7,17-21}$ Moreover, it has been communicated at least once in Africa ${ }^{22}$ and it has been found in ticks in South America. ${ }^{23,24}$ Despite the relatively small sample size and the unicenter nature of the study, data showed in this study add further evidence that distribution of HGA is widespread.

In line with previous reports, possible co-infections were detected in more than one third of cases in our cohort. ${ }^{19-21}$ Physicians must be aware of possible co-infections not only with tick-borne microorganisms but also with mosquito-transmitted arboviruses.

Although most cases presented a self-limited evolution, effective antibiotic treatment against intracellular bacteria such as doxycycline should be considered in travellers with undifferentiated fever presenting with severe disease, after excluding malaria and, especially, if they had visited rural areas and/or presented with cytopenias. ${ }^{5,20,21,24}$

In conclusion, HGA should be included in the differential diagnosis of returning travellers with fever and no clear source of infection. Antibiotic treatment such as doxycycline should be considered in travellers with undifferentiated fever presenting with severe disease, after discarding malaria and, especially, if they had visited rural areas and/or presented with cytopenias. Physicians must be aware of possible co-infections with other tick-borne microorganisms and mosquito-transmitted arboviruses.

\section{Supplementary data}

Supplementary data are available at JTM online.

\section{Conflict of interests}

The authors declare no conflict of interests.

\section{Acknowledgements}

This study was awarded with 2017-2018 International Society of Travel Medicine (ISTM) Research Award.

\section{References}

1. Sanders JM, Monogue ML, Jodlowski TZ, Cutrell JB. Pharmacologic treatments for coronavirus disease 2019 (COVID-19): a review. JAMA 2020; 323:1824-36.

2. Hagmann SH, Han PV, Stauffer WM et al. Travel-associated disease among US residents visiting US GeoSentinel clinics after return from international travel. Fam Pract 2014; 31:678-87.
3. Buss I, Genton B, D'Acremont V. Aetiology of fever in returning travellers and migrants: a systematic review and meta-analysis. $J$ Travel Med 2020; 27:taaa207. doi: 10.1093/jtm/taaa207 PMID: 33146395; PMCID: PMC7665639.

4. Bottieau E, Clerinx J, Schrooten W et al. Etiology and outcome of fever after a stay in the tropics. Arch Intern Med 2006; 166:1642-8.

5. Mayxay M, Castonguay-Vanier J, Chansamouth V et al. Causes of non-malarial fever in Laos: a prospective study. Lancet Glob Health 2013; 1:e46-54.

6. Brouqui P, Bacellar F, Baranton G et al. Guidelines for the diagnosis of tick-borne bacterial diseases in Europe. Clin Microbiol Infect 2014; 10:1108-32.

7. Jin H, Wei F, Liu Q, Qian J. Epidemiology and control of human granulocytic anaplasmosis: a systematic review. Vector Borne Zoonotic Dis 2012; 12:269-74.

8. Blanco JR, Oteo JA. Human granulocytic ehrlichiosis in Europe. Clin Microbiol Infect 2002; 8:763-72.

9. Jensenius M, Davis X, von Sonnenburg F et al. GeoSentinel surveillance network. Multicenter GeoSentinel analysis of rickettsial diseases in international travelers, 1996-2008. Emerg Infect Dis 2009; 15:1791-8.

10. Nitzan O, Blum A, Marva E et al. Case report: infectious diseases in pilgrims visiting the holy land. Am J Trop Med Hyg 2017; 97: 611-4.

11. Courtney JW, Kostelnik LM, Zeidner NS, Massung RF. Multiplex real-time PCR for detection of anaplasma phagocytophilum and Borrelia burgdorferi. J Clin Microbiol 2004; 42:3164-8. doi: 10.1128/JCM.42.7.3164-3168.2004.

12. Silaghi C, Santos AS, Gomes J et al. Guidelines for the direct detection of Anaplasma spp. in diagnosis and epidemiological studies. Vector Borne Zoonotic Dis 2017; 17:12-22.

13. Zeidner NS, Burkot TR, Massung R. Transmission of the agent of human granulocytic Ehrlichiosis by Ixodes spinipalpis ticks: evidence of an enzootic cycle of dual infection with Borrelia burgdorferi in northern Colorado. J Infect Dis 2000; 182: 616-9.

14. Phommasone $\mathrm{K}$, Paris $\mathrm{DH}$, Anantatat $\mathrm{T}$ et al. Concurrent infection with murine typhus and scrub typhus in southern Laos-the mixed and the unmixed. PLoS Negl Trop Dis 2013; 7:e2163. doi: 10.1371/journal.pntd.0002163.

15. The potential impact of health service disruptions on the burden of malaria: a modelling analysis for countries in sub-Saharan Africa. Geneva: World Health Organization, 2020, Licence: CC BY-NC-SA 3.0 IGO.

16. Zammarchi L, Gobbi F, Angheben A et al. Schistosomiasis, strongyloidiasis and Chagas disease: the leading imported neglected tropical diseases in Italy. J Travel Med 2020; 27:taz100. doi: 10.1093/jtm/taz100.

17. Blacksell SD, Kantipong P, Watthanaworawit W et al. Underrecognized arthropod-borne and zoonotic pathogens in northern and northwestern Thailand: serological evidence and opportunities for awareness. Vector Borne Zoonotic Dis 2015; 15:285-90. doi: 10.1089/vbz.2015.1776.

18. Koh FX, Kho KL, Kisomi MG et al. Ehrlichia and Anaplasma infections: serological evidence and tick surveillance in peninsular Malaysia. J Med Entomol 2018; 55:269-76. doi: 10.1093/jme/tjx204.

19. Nooroong P, Trinachartvanit W, Baimai V, Ahantarig A. Phylogenetic studies of bacteria (Rickettsia, Coxiella, and Anaplasma) in Amblyomma and Dermacentor ticks in Thailand and their coinfection. Ticks Tick Borne Dis 2018; 9:963-71.

20. Heo DH, Hwang JH, Choi SH et al. Recent increase of human granulocytic anaplasmosis and co-infection with scrub typhus or 
Korean hemorrhagic fever with renal syndrome in Korea. J Korean Med Sci 2019; 34:e87. doi: 10.3346/jkms.2019.34.e87.

21. Le-Viet N, Le VN, Chung $\mathrm{H}$ et al. Prospective case-control analysis of the aetiologies of acute undifferentiated fever in Vietnam. Emerg Microbes Infect 2019; 8:339-52.

22. Elhamiani Khatat S, Sahibi H, Hing $\mathrm{M}$ et al. Human exposure to Anaplasma phagocytophilum in two cities of northwestern Morocco. PLoS One 2016; 11:e160880.
23. Pesquera C, Portillo A, Palomar AM, Oteo JA. Investigation of tick-borne bacteria (Rickettsia spp., Anaplasma spp., Ehrlichia spp. and Borrelia spp.) in ticks collected from Andean tapirs, cattle and vegetation from a protected area in Ecuador. Parasit Vectors 2015; 8:46.

24. Licona-Enríquez J, Delgado-de la Mora J, Alvarez-Hernandez G. Fatal case of co-infected of rickettsiosis and dengue virus in Mexico. Rev Med Inst Mex Seguro Soc 2018; 56:320-2. 